\title{
IMPLICACIONES DE LOS PRINCIPIOS PRECAUTORIO Y PREVENTIVO PARA EL DERECHO A UN AMBIENTE SANO EN AMÉRICA LATINA
}

IMPLICATIONS OF THE PRECAUTIONARY AND PREVENTIVE PRINCIPLES FOR THE RIGHT TO A HEALTHY ENVIRONMENT IN LATIN AMERICA

\section{IMPLICAÇÕES DOS PRINCÍPIOS DE PRECAUÇÃO E PREVENÇÃO PARA O DIREITO A UM MEIO AMBIENTE SAUDÁVEL NA AMÉRICA LATINA}

\section{Aleida Azamar Alonso ${ }^{1}$}

\section{Resumen}

Este trabajo tiene como objetivo analizar las implicaciones de los principios de precaución y prevención para la regulación ambiental de la minería y el fracking en América Latina para garantizar el acceso a un ambiente sano. La metodología empleada consistió en revisión documental de distintas fuentes sobre dichas actividades y sus consecuencias, así como en el análisis de la parte legislativa sobre los principios de precaución y de prevención. Los resultados indican que la forma en que dichas herramientas normativas se han aplicado carece de efectos en el largo plazo para la prevención de otros riesgos. Como conclusión, se estima que la debilidad institucional y la dependencia de estas actividades en la región es lo que provocan la falta de políticas integrales que garanticen bienestar socioambiental en el largo plazo.

Palabras clave: Regulación, riesgos, minería, fracking.

\begin{abstract}
This paper aims to analyze the implications of the precautionary and prevention principles as used in the mining and fracking environmental regulation in Latin America in order to guarantee access to a healthy environment. The methodology used consisted of a document review of
\end{abstract}

Doi: https://doi.org/10.15359/eys.26-59.2

Recibido: 11-09-2020. Reenvíos: 04-11-2020, 16-11-2020, 25-01-2021. Aceptado: 30-03-2021. Publicado: 09-04-2021.

1 Profesora Investigadora del Departamentode Producción Económica, Universidad Autónoma Metropolitana, Xochimilco. Correo: gioconda15@gmail.com, ORCID: https://orcid.org/0000-0002-7915-7611

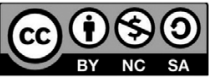


different sources on these activities and their consequences, as well as an analysis of legislation regarding the precaution and prevention principles.

Results indicate that the way in which these regulatory tools have been applied has no long-term effects for the prevention of other risks. It is concluded that both institutional weakness and dependence on these activities in the region is what causes the lack of comprehensive policies that guarantee socio-environmental well-being in the long term.

Keywords: regulation, risks, mining, fracking.

\section{Resumo}

Este trabalho tem como objetivo analisar as implicações dos princípios da precaução e prevenção para o regulamento ambiental da mineração e do fraturamento hidráulico na América Latina com o fim de garantir o acesso a um meio ambiente saudável. A metodologia utilizada consistiu na revisão documental de diferentes fontes sobre essas atividades e suas consequências, bem como na análise da parte legislativa sobre os princípios da precaução e prevenção. Os resultados indicam que a forma como essas ferramentas normativas têm sido aplicadas não tem efeitos de longo prazo para a prevenção de outros riscos. Em conclusão, estima-se que a fragilidade institucional e a dependência dessas atividades na região são as responsáveis pela falta de políticas integrais que garantam o bemestar socioambiental de longo prazo.

Palavras-chave: regulamento, riscos, mineração, fraturamento hidráulico.

\section{Introducción}

El objetivo de este trabajo es realizar una revisión de los principios de prevención y precaución en relación con el derecho a un ambiente sano en América Latina, con la finalidad de comprender sus alcances, limitantes y espacios de mejora. Para ello se utilizó un método de análisis documental e histórico en el que se identificaron los elementos conceptuales que constituyen y fundamentan la estructura de los principios: precautorio y de prevención con diferentes ejemplos de las prácticas de minería y fracking en la región Latinoamérica, en los cuales se vieron afectados diversas poblaciones y el ecosistema, señalando los casos, las medidas y la valoración al respecto. También se elaboró un acercamiento a los fundamentos prácticos y teóricos de los principios legislativos, por lo que se consideraron perspectivas clásicas y modernas respecto al complejo entramado de estos en la normativa regional.

El artículo cuenta con tres apartados. En el primero, se explica la concepción del concepto del riesgo como elemento interpretativo de la realidad y su implicación en las políticas del Estado y el mercado. Por otra parte, en la segunda sección, se discuten algunos aspectos sobre los principios de precaución y prevención, así como las formas normativas que toman al ser aplicados. En el tercer apartado se señalan tres casos 
particulares que también suceden en otros países de la región. Finalmente, se presentan las conclusiones.

\section{Sobre el concepto del riesgo}

Los seres humanos no suelen concebir a las actividades productivas en su justa medida de riesgo debido a que no existen las herramientas para poder calcularlos con exactitud. La forma en que se determina el nivel y el alcance de estos riesgos generalmente parte de una comprensión técnico-científica, sin dejar lugar a otra forma de interpretación. Esta manera de abordar la cuestión es la que comúnmente se emplea en las instituciones gubernamentales y en los sectores privados para calcular los costos-beneficios de sus acciones. Además, esta también se ocupa en el lenguaje académico de algunas ciencias centradas en las tendencias probabilísticas de diversos indicadores cuantitativos.

Se trata de una posición que se enfoca en la racionalidad de las acciones individuales, pues a partir de "estudiar la forma en que las personas perciben, analizan y toman decisiones sobre diferentes situaciones" (Farràs, Bosch y Torrente, 2008:2) se crean modelos predictivos de relaciones entre alcances, dimensiones y la manera en que los expertos (Estado y aquellos autorizados por este último) deben resolver el problema dando lugar a la creación de normatividades estandarizadas, limitadas en su propia concepción. Esta perspectiva permite establecer dinámicas de trabajo focalizadas, pero también tiene como limitación adaptarse a contextos en los que aspectos culturales, sociales y de otra índole puedan tener mayor peso en la toma de decisiones. Debido a dichas limitaciones, otros autores han planteado enfoques más diversos para discutir la cuestión del riesgo, algunos de los cuales se recuperan a continuación.

Una de las limitaciones más importantes es la planteada por Douglas (1987), ya que en su abordaje antropológico destaca que la perspectiva técnico-científica comentada se sesgaba entorno al paradigma social imperante, pues el riesgo es una categoría social construida por el colectivo enmarcado en una pauta cultural subjetiva (Zinn, 2008). Es decir, los daños próximos de una determinada acción no necesariamente son un riesgo por sí mismos, sino que deben mediarse ante un parámetro ético y moral que solamente se considerarán un problema si afectan el orden imperante, pues, de otra manera, la sociedad en su conjunto estaría constantemente detenida por las múltiples amenazas que existen y que son inevitables. Esta forma de pensamiento es la que Douglas (1996) denomina "inmunidad subjetiva" (p. 97); además, esta es la que condiciona a las personas a subestimar situaciones peligrosas.

Considerando el abordaje de Douglas, es posible señalar que un esquema normativo muy rígido en cuanto a la posibilidad de adaptarse a otros contextos condiciona la percepción social del riesgo a intereses específicos. Por ejemplo, ante la creación de una regulación para beneficiar a un sector productivo nocivo para la naturaleza, es posible que una parte de la sociedad crea que existe una mayor amenaza al corregir el sesgo regulador mencionado en contraste con los propios efectos negativos de sus actividades, los cuales se asumen como aceptables debido a la norma en ejecución. 
Por otra parte, resulta interesante considerar la posición de Giddens ${ }^{2}$ (2001) respecto al hecho de que el desarrollo industrial y el crecimiento económico actual generan, por sí mismos, riesgos, cuyas características relevantes son su modernidad y colectividad. Para el autor, no se trata de que la sociedad se encuentre en una época más peligrosa que las anteriores, sino que resulta más fácil ahora trazar las condiciones que desatan situaciones potencialmente peligrosas en cuanto estas se derivan de las acciones humanas y son de escala planetaria.

Giddens (2001) no rechaza la posibilidad de que todavía existan fenómenos naturales ajenos a las acciones de las personas, sino que sugiere que los efectos de mayor relevancia pueden ser resultado del impacto que tienen las actividades antropogénicas en la actualidad, considerando que estos son "riesgos manufacturados". Es otras palabras, sucede con mayor frecuencia un problema socioambiental de gran escala producto de la forma de vida colectiva moderna, en comparación con, por ejemplo, un desastre natural.

Dicha afirmación debe realizarse con algunos matices, pues, si bien sí suceden múltiples desastres ambientales derivados de una mala práctica productiva, estos no tienen la escala y magnitud de un fenómeno meteorológico grave como puede ser un huracán, una explosión volcánica, etc. Por otra parte, la amenaza de las actividades antropogénicas puede provocar otras formas de daños a largo plazo que van desde pequeñas modificaciones territoriales hasta la amenaza a la capacidad de la sostenibilidad de la vida en un espacio determinado.

La cuestión de fondo reside en la forma en la que suceden estos eventos, ya que una situación que implique un desastre natural es, generalmente, producto de elementos fuera del control humano. Mientras que un "accidente" y sus consecuencias se tratan de circunstancias en las que puede influir activamente la sociedad para evitar que se repitan $y$, en pro de este control, se desarrollan varias formas de regulación que disminuyen la probabilidad de que sucedan, aunque esto tenga efectos, en algunos casos, no deseables.

A continuación, se discuten los enfoques respecto a las herramientas legislativas empleadas para prevenir o disminuir los riesgos comentados anteriormente. Cabe señalar que, en este trabajo, se mencionan casos sobre la minería y el fracking en América Latina, por lo cual los argumentos que se esgrimen a continuación se fundan en la identificación de fenómenos asociados a estos sectores.

\section{Normatividad de prevención del riesgo socioambiental}

La normatividad que rige la práctica de actividades potencialmente dañinas para la naturaleza y, por extensión, para el ser humano, se encuadra en el marco de la política y el derecho ambiental. Estas últimas son herramientas para regular la forma en que se utilizan los bienes naturales para lograr un equilibrio entre las necesidades humanas, las

2 Cuestión que también recupera Beck, aunque este autor se centra en los procesos de transformación institucional y social proponiendo los conceptos de Sociedad del riesgo y modernidad reflexiva ante el inevitable avance de la industrialización moderna. Por lo anterior, y en función de la utilidad para este trabajo, se hace referencia a la propuesta de Giddens (2001). 
demandas industriales y los ciclos de reproducción ecosistémicos (Andaluz, 2006). De esta manera, surgen los principios precautorio y preventivo, entre muchos otros, que grosso modo se emplean para limitar los efectos negativos de las actividades productivas que pueden vulnerar el equilibrio ecológico y social.

Resulta evidente que la ejecución de estos mecanismos se adhiere a la realidad local de cada país donde se lleva a cabo, pero confluyen en el interés de mantener cierto nivel de protección a la naturaleza y también para las personas. Por ello, la Organización de las Naciones Unidas (ONU) y otras instituciones internacionales, han sugerido que, a discreción de cada país, algunas de estas regulaciones sean consideradas como parte de sus componentes legislativos en materia ambiental.

A continuación, se menciona la forma en que los principios precautorio y preventivo surgieron en Alemania y Estados Unidos a mediados del siglo pasado, adoptándose después a nivel internacional con un enfoque diferente y más limitado del que originalmente tuvieron.

\subsection{Principio precautorio}

Con el propósito de enfrentar algunos de los posibles riesgos generados por los procesos industriales de las diferentes actividades de aprovechamiento de los bienes naturales, existe el "principio precautorio". Dicho principio tiene su origen en la práctica del derecho y la protección ambiental alemana de la década de los setenta (Cafferatta, 2004), así como en el Programa del Medio Ambiente de las Naciones Unidas de 1972 en el que se recomienda a todas las naciones adoptar el principio de precaución para la eliminación y disminución de la polución marina. Después, en la Reunión Ministerial de Bremen de la Conferencia de Protección del Mar del Norte (1984) se describe de forma más amplia. También, ha sido mencionado en el Protocolo de la Capa de Ozono en 1985; así como en la Segunda Delación del Mar del Norte de 1987 y, en al menos otros diez documentos internacionales más en los que se destaca la importancia de adoptar dicho principio.

Este concepto adquirió un mayor nivel de relevancia ${ }^{3}$ cuando fue presentado en la Declaración de Río de Janeiro, durante la Conferencia de la ONU sobre la situación del ambiente y el nivel de desarrollo en el año de 1992, señalando lo siguiente:

Principio 15: Con el fin de proteger el medio ambiente, los Estados deberán aplicar ampliamente el criterio de precaución conforme a sus capacidades. Cuando exista peligro de daño grave e irreversible, la falta de certeza científica absoluta no deberá utilizarse como razón para postergar la adopción de medidas eficaces en función de los costos para impedir la degradación del medio ambiente. (ONU, 1992)

3 Cabe destacar que en el Tratado Maastricht de la Unión Europea de 1992 dicho principio se adoptó como una política comunitaria que sigue las prácticas de cautela y acción preventiva, mismas que están vigentes hasta la actualidad.

Luis Guillermo Davinson Pacheco - Ulises Armando Asenjo Ramírez 
El principio precautorio se justifica en base a dos particularidades específicas: la primera, que algunas actividades de las que se desconocen a ciencia cierta sus efectos pueden tener impactos graves e irreversibles, tanto para los seres humanos como para la naturaleza; la segunda, es el supuesto de "certeza de la incertidumbre", el cual se refiere a la existencia de que pueda suceder una improbabilidad en cualquier sistema natural debido a la interferencia de las actividades humanas.

De manera que este principio de protección socioambiental considera que las actividades productivas, por su propia existencia, generan riesgos ecosistémicos como resultado de su práctica, los cuales no pueden ser identificados, analizados y estudiados en la misma velocidad a la que son implementados, por lo que se debe establecer una regulación que proteja tanto a la naturaleza como al ser humano. No obstante, dicho principio solamente puede ser aplicado en virtud de los siguientes aspectos:

1. Existe incertidumbre científica apreciable o incluso ignorancia acerca del daño previsto; 2. Existen hipótesis (o modelos) de posibles daños que sean científicamente razonables ; 3 . Resulta de momento imposible reducir la incertidumbre ; 4 . el daño potencial es suficientemente grave o incluso irreversible; 5 . cualquier medida eficaz adoptada ulteriormente para contrarrestarlo resultaría mucho más difícil u onerosa. (Comisión Mundial de Ética del Conocimiento Científico y la Tecnología (COMEST), 2005:49)

Así, la aplicación de este principio se basa en dar el beneficio de la duda a favor de la naturaleza en tanto exista la noción de que esta última puede salir perjudicada, por lo que las medidas que se tomen al respecto deben estar en proporcionalidad con dicha duda. Por otra parte, también debe señalarse que este principio se ha convertido en parte del Derecho internacional consuetudinario por lo extendido de su práctica y su uso para denunciar acciones de países que no guardan el interés suficiente para actuar de forma cautelar ante riesgos inminentes o desconocidos (Trouwborst, 2007).

No obstante, como aparece en la referencia a la Declaración de Río de 1992, la aplicación del principio precautorio se hará conforme a las capacidades locales, lo que es una limitante que puede ser aprovechada por interesados en estas actividades para realizar proyectos que se beneficien de las áreas grises en las que las administraciones públicas no cuenten con suficientes datos para realizar una estimación fiable de los posibles daños que se pueden provocar.

El principio de precaución es una herramienta fundamentada en la incertidumbre o desconocimiento de las diversas formas en las que pueden impactar las actividades productivas a la salud humana o al bienestar ambiental. Evidentemente, se requieren otros mecanismos cuando se tiene conocimiento de los daños que se asume como posibles en virtud del ejercicio de prácticas destructivas a nivel socioambiental. 


\subsection{Principio de prevención}

En contraposición al principio precautorio, la lógica de la prevención se atañe más al sentido común que a la intuición y observación. Esto se debe a que asume que existen determinados niveles de riesgo que pueden irse regulando en función de las necesidades, intereses y capacidades de control para evitar que suceda un problema. Se podría considerar que esta cuestión tiene lugar por cuanto viene a significar la base de la Ley y el derecho ambiental en casi todo el mundo. Básicamente esta es la forma en la que se abordan los riesgos que ya han sido comprobados científicamente, por lo que se deben aplicar medidas adecuadas y dirigidas para atenuar los efectos negativos inmediatos y futuros.

El "principio preventivo" nace en 1941 a raíz de un reclamo en los tribunales estadounidenses a un proceso industrial que se presentó en Canadá, debido a que se estaban emitiendo gases tóxicos desde finales del siglo XIX, hecho que provocaba enfermedades y desgaste territorial en suelo estadounidense. Por ello, se establece una sentencia condenatoria que señala que los Estados son responsables de aprovechar sus bienes naturales sin que ello implique algún daño ambiental más allá de sus límites jurisdiccionales. Esta sentencia fue adoptada y adaptada después al principio 21 del Informe de la Conferencia de las Naciones Unidas sobre el Medio, de 1972. Más adelante, se adopta como el principio número 2 de la Declaración de Río de 1992, reforzando así su carácter consuetudinario, lo que quedó plasmado en la Declaración de Río de 2012.

Es así como el principio preventivo se convirtió en un elemento activo y fundamental para limitar cualquier posible riesgo o perjuicio socioambiental por alguna práctica productiva sin importar su origen o interés final. Actualmente, se puede considerar que se trata de una herramienta destinada a minimizar la contaminación ambiental (Kiss y Shelton, 2007).

El problema es que su aplicación es interna y no transfronteriza, por lo que carece del mismo rigor de aplicación internacional y no se encuentra contenida en ninguna declaratoria o Convenio internacional, sino que es parte de los procesos normativos locales que se han adaptado a esta necesidad práctica preventiva sin alcanzar por sí misma la condición de derecho internacional consuetudinario (Lefeber, 1996; Sands, 2018).

Debido a que hay pocas o nulas sentencias legislativas internacionales en favor de agraviados que reclamen la falta de aplicación del principio preventivo, contrario a lo que sucede con el de precaución, es difícil utilizar esta herramienta para una denuncia internacional de comunidades o poblaciones que se vean amenazadas por proyectos locales o extranjeros en los que el principio de precaución no puede aplicarse y el de prevención no se llevó a cabo de forma adecuada. También, debido a la misma razón, depende de la legislación local y de los procesos normativos vigentes a nivel regional mediante los cuales se puede desconocer o rechazar esta práctica.

Por ejemplo, en América Latina, la extracción de minerales e hidrocarburos se ha convertido en parte fundamental de su estrategia económica, por lo que existe una gran 
dependencia de los ingresos tributarios y de inversión para la explotación intensiva de estos recursos (Azamar, 2018), lo cual pone en predicamento la posibilidad de ejecutar los principios comentados para garantizar el bienestar socioambiental de la región. Por ello, a continuación, se discute la capacidad de garantizar el derecho a un ambiente sano, para un escenario complejo como lo es América Latina donde las instituciones de gobierno y las necesidades locales dificultan la aplicación de este tipo de regulaciones.

\section{Marco y alcance de los principios de prevención y precaución para garantizar un ambiente sano ante las actividades extractivas}

A la par del desarrollo de estos mecanismos legislativos mencionados, también ha surgido la discusión internacional sobre el alcance de los derechos humanos fundamentales ${ }^{4}$, siendo el más evidente e importante el derecho a la vida. A fin de poder cumplir con este último, se deben garantizar varios aspectos: alimentación saludable, agua potable, techo $y$, por supuesto, un ambiente sano ${ }^{5}$.

Sin embargo, hay actividades productivas esenciales para el sistema económico moderno que pueden afectar la práctica de estos derechos. Por ejemplo, la minería, el fracking y la extracción de hidrocarburos, las cuales, en su ejecución, conducen a la contaminación del agua y el subsuelo por el uso de químicos y por la dificultad de impedir su filtración, por lo que también empobrecen la calidad del aire por sus emanaciones y vapores; asimismo, provocan enfermedades respiratorias y otros padecimientos en la piel derivados de los procesos de trabajo en los que existen elementos que todavía son desconocidos para poder controlar su nivel de riesgo (Pérez et al. 2016).

El problema surge cuando la capacidad de un Estado para contar con financiamiento para cumplir con estas responsabilidades depende de dichas actividades productivas. En este escenario, por una parte, la práctica de estas últimas violenta los derechos comentados $y$, por otro lado, en caso de ejecutarse, se puede debilitar la fortaleza institucional por la falta de ingresos, de manera que se pueden afectar otros derechos universales y fundamentales.

La ejecución de los principios preventivo y precautorio puede conducir a situaciones indeseadas si se aplican de forma irreflexiva prohibiendo este tipo de proyectos sin apoyarse de otras herramientas de trabajo colectivo para proteger y beneficiar a quienes dependen de las mismas; pero, un esfuerzo tibio para regularlas deja a la sociedad expuesta al interés económico. Sobre todo, si se considera que las empresas mineras y de fracking tienen un alto poder de influencia política y económica debido a la importante

4 De acuerdo con Arroyo (2012) los derechos humanos son aquellas prerrogativas que deberían ser aplicables a cualquier persona solamente por existir independientemente del lugar en el que se encuentre, mientras que los derechos fundamentales son los que se reconocen en una Constitución política nacional. Es decir, no todos los derechos son universales, pues en gran medida pueden depender de la condición política de los individuos.

5 Hasta el año 2020 en la mayor parte de América Latina el derecho a un ambiente saludable era una cuestión más o menos opaca o de interés secundario. En el mes de noviembre de ese año que se da a conocer que 11 países firmaron el Acuerdo de Escazú garantizando que los Estados deben cumplir con el interés de proteger este aspecto de forma activa y prioritaria. 
base tributaria de estas actividades, así como a los millonarios niveles de inversión que ingresan debido a este tipo de proyectos.

De este modo, no resulta sorpresivo que estos actores privados quieran influir en las acciones del Estado, especialmente en aquellas que afectan sus capacidades productivas o que podrían ser determinantes para limitar sus beneficios (Aidun y Giunta, 2019; Campanini, Gandarillas y Gudynas, 2019; Svampa, 2019).

Al respecto, resulta adecuado resaltar que existen múltiples casos documentados de corrupción estatal en varios países de América Latina en donde los empresarios de los sectores comentados han influido para facilitar permisos de exploración-explotación, así como para limitar las revisiones en los casos de abuso y violencia contra la población que rechaza estas actividades (Aidun y Giunta, 2019; Azamar, 2018; Campanini, Gandarillas y Gudynas, 2019).

El hecho de que en América Latina sucedan la mayor parte de crímenes mortales en el mundo contra los defensores ambientales que rechazan estas actividades (Azamar y Olivera, 2021) y que, además, sean precisamente la minería y el fracking las actividades que tienen más conflictos socioambientales en la región en la actualidad (Zaremberg y Guarneros, 2019) como resultado de un deficiente manejo regulador por parte del Estado, permite suponer que existen serias deficiencias en la forma de aplicar las normas de protección ambiental y social, las cuales, se supone, deberían estar guiadas por el principio de precaución y por el de prevención.

En consonancia con lo comentado, a continuación, se exponen, mediante la Tabla 1, algunos casos sobre riesgos de la minería y fracking en América Latina, así como acuerdos internacionales para prevenir la falta de protección socioambiental.

\section{Tabla 1}

Riesgos de incertidumbre, formas de prevención y precaución para la minería y el fracking en América Latina

\begin{tabular}{|c|c|c|c|c|c|}
\hline Situación & $\begin{array}{c}\text { Estado y } \\
\text { fecha de los } \\
\text { conocimientos }\end{array}$ & Caso & $\begin{array}{l}\text { Tipo de } \\
\text { medida }\end{array}$ & $\begin{array}{l}\text { Ejemplos de } \\
\text { acciones }\end{array}$ & $\begin{array}{c}\text { Garantía de } \\
\text { un ambiente } \\
\text { saludable }\end{array}$ \\
\hline Riesgo & $\begin{array}{l}\text { Impactos y } \\
\text { probabilidades } \\
\text { conocidas }\end{array}$ & $\begin{array}{l}\text { Contaminación } \\
\text { en todos los tipos } \\
\text { de ecosistemas } \\
\text { por el uso de } \\
\text { una mezcla } \\
\text { de químicos } \\
\text { (mercurio, } \\
\text { ácidos, etc.) } \\
\text { potencialmente } \\
\text { dañinos }\end{array}$ & Prevención & $\begin{array}{l}\text { Compromiso } \\
\text { político de } \\
\text { prohibición } \\
\text { de técnicas } \\
\text { de fracking en } \\
\text { Colombia y en } \\
\text { México }\end{array}$ & $\begin{array}{l}\text { Parcialmente, } \\
\text { no hay evidencia } \\
\text { contundente de } \\
\text { que realmente se } \\
\text { hayan detenido } \\
\text { estas actividades } \\
\text { y existe la } \\
\text { posibilidad de } \\
\text { que se estén } \\
\text { ejecutando de } \\
\text { manera opaca }\end{array}$ \\
\hline
\end{tabular}




\begin{tabular}{|c|c|c|c|c|c|}
\hline Situación & $\begin{array}{c}\text { Estado y } \\
\text { fecha de los } \\
\text { conocimientos }\end{array}$ & Caso & $\begin{array}{l}\text { Tipo de } \\
\text { medida }\end{array}$ & $\begin{array}{l}\text { Ejemplos de } \\
\text { acciones }\end{array}$ & $\begin{array}{c}\text { Garantía de } \\
\text { un ambiente } \\
\text { saludable }\end{array}$ \\
\hline Incertidumbre & $\begin{array}{l}\text { Impactos y } \\
\text { probabilidades } \\
\text { parcialmente } \\
\text { desconocidas }\end{array}$ & $\begin{array}{l}\text { La falta de } \\
\text { información } \\
\text { sobre } \\
\text { mecanismos } \\
\text { de defensa } \\
\text { institucional } \\
\text { ante casos de } \\
\text { explotación } \\
\text { ambiental } \\
\text { violenta e } \\
\text { intensiva }\end{array}$ & $\begin{array}{l}\text { Prevención } \\
\text { cautelar }\end{array}$ & $\begin{array}{l}\text { Acuerdo de } \\
\text { Escazú }\end{array}$ & $\begin{array}{l}\text { Parcialmente, si } \\
\text { bien el acuerdo } \\
\text { es vinculatorio, } \\
\text { no integra a } \\
\text { todos los países } \\
\text { de América Latina } \\
\text { y deja abierta } \\
\text { la puerta a la } \\
\text { interpretación } \\
\text { de cada país } \\
\text { en el tema de } \\
\text { protección } \\
\text { ambiental }\end{array}$ \\
\hline Ignorancia & $\begin{array}{l}\text { Impactos y } \\
\text { probabilidades } \\
\text { desconocidas }\end{array}$ & $\begin{array}{l}\text { El uso y } \\
\text { contaminación } \\
\text { del agua, } \\
\text { así como la } \\
\text { depredación de } \\
\text { los bosques para } \\
\text { la explotación de } \\
\text { recursos mineros } \\
\text { metálicos }\end{array}$ & Precaución & $\begin{array}{l}\text { Las actividades } \\
\text { de minería } \\
\text { metálica son } \\
\text { prohibidas } \\
\text { totalmente como } \\
\text { en el caso de El } \\
\text { Salvador y Costa } \\
\text { Rica }\end{array}$ & $\begin{array}{l}\text { No, la falta de } \\
\text { programas de } \\
\text { apoyo a las } \\
\text { comunidades } \\
\text { dependientes } \\
\text { de la minería ha } \\
\text { provocado un } \\
\text { mercado ilegal } \\
\text { más dañino y } \\
\text { riesgoso }\end{array}$ \\
\hline
\end{tabular}

Fuente: Elaboración propia, 2020.

Como es posible observar en la Tabla 1, existen diversas formas de acercarse a los impactos de la minería y del fracking, partiendo de mecanismos de prevención y precaución. La intención de este cuadro es exponer el orden jerárquico en el que se ha abordado el riesgo de estas actividades en algunos lugares de América Latina y señalar si las mismas han tenido un efecto potencialmente efectivo para garantizar el acceso a un ambiente sano.

Los acuerdos legislativos internacionales son determinaciones jurídicas ante problemas conocidos, pero su ejecución queda en manos de las autoridades que pueden ser o no competentes en la aplicación de estos, hecho que resulta especialmente notable con el Acuerdo de Escazú, el cual, en su artículo 3, señala que este se guiará por los principios preventivo y precautorio, entre algunos otros, que, a pesar de estar presentes de forma implícita en varias normatividades de la región, en muchos casos no forman parte central de sus estructuras constitucionales (Romero, 2017).

La prohibición de una práctica tampoco es la forma más eficiente para abordar un problema, por lo menos no en América Latina; si bien existe evidencia de que más de 15 países en el mundo han prohibido la minería y el fracking de forma parcial o absoluta con resultados positivos a nivel socioambiental (Aidun y Giunta, 2019), en esta región las 
instituciones públicas no son del todo transparentes con respecto a esta situación ${ }^{6}$, por lo cual existen dudas más que razonables sobre su actuación e interés final.

Por ejemplo, la actuación de Colombia sobre el fracking responde tanto a su Ley 99 de 1993 respecto al principio precautorio en el que existen incertidumbres sobre los riesgos de estas actividades, pero la evaluación de éstas depende de cabilderos empresariales del fracking. De manera que la aplicación de este principio es más un proceso político en el que interviene el riesgo manufacturado, pues a la población se le ha convencido de que existe un mayor peligro de pobreza por no llevar a cabo esta actividad que por su ejecución, comprando la voluntad local (Pozzi, López y Gálvez, 2019).

En cuanto a México, el tema sobre la prohibición al fracking fue una promesa hecha por el actual presidente de la República durante su campaña, lo que además formó parte de su plan de trabajo sexenal. Sin embargo, hoy se sabe que la principal empresa petrolera del país utiliza esta práctica de forma constante desde hace décadas sin que exista evidencia de que pueda cambiar en un corto periodo, a pesar de que esto contraviene lo dicho públicamente por el presidente (Pozzi, López y Gálvez, 2019); asimismo, violenta el artículo 4to de la Constitución, en el cual se garantiza el acceso a un ambiente sano. De igual forma, debe destacarse que, a pesar de que el principio precautorio es vinculante para este país, su normatividad no deja en claro que exista un mecanismo para contrarrestar de forma efectiva un riesgo grave ante la incertidumbre científica (Romero, 2017).

En el caso de Costa Rica, la prohibición de la minería a cielo abierto en el año 2010 respondió principalmente al rechazo social por el daño a los bosques del país, también ante los efectos ambientales desconocidos que se encuentran contenidos en su normatividad, lo que sí reconoce el principio precautorio. Sin embargo, esta cuestión ha tenido como consecuencia que varios grupos de mineros ilegales se aprovechen de las vetas ya descubiertas y generen situaciones de riesgo ambiental por la falta de medidas de protección social, porque amenazan a la población que rechaza esta práctica. Dicha situación ha provocado que, en la actualidad, se mantenga viva la discusión política sobre el tema de reactivar esta práctica extractiva para contrarrestar dicha situación, a pesar de que esto contraviene su reconocimiento al derecho a un ambiente sano y ecológicamente equilibrado de forma constitucional.

En El Salvador, la situación es similar a la de Costa Rica; sin embargo, en este país no se contempla el principio precautorio en su normatividad, por lo que la prohibición de la minería metálica fue resultado de la presión social y comunitaria que obedecía a una percepción local del riesgo ante la evidencia ya conocida. En los últimos meses esta nación avanza hacia una apertura del debate sobre reactivar esta actividad ante las dificultades económicas que atraviesan actualmente sus habitantes. La falta de

6 Esto se puede considerar especialmente a la luz de los casos de violencia comentados anteriormente, pero a ello se puede sumar que ningún país en la región ha creado un mecanismo para el acceso a información sobre las violaciones sistemáticas a los derechos socioambientales y a las acciones por parte del Estado para frenarlas, por lo que el Acuerdo de Escazú es fundamental para transitar hacia un escenario más transparente. 
herramientas normativas y políticas ambientales integrales, así como la ausencia de un derecho constitucional explícito a un ambiente sano, dificultan enfrentar este tipo de escenarios para quienes rechazan estas actividades extractivas que en este caso llegaron a contaminar uno de los ríos más importantes de este país.

\section{Conclusiones}

En los países en vías de desarrollo, existe una limitada capacidad institucional para enfrentar los retos que supone comprender el impacto negativo socioambiental de la minería y del fracking. Esta debilidad provoca falta de conocimiento o información para demostrar plausiblemente que existe un riesgo por el uso de químicos o por la realización de ciertas técnicas de extracción. A fin de enfrentar esta cuestión, algunas regulaciones socioambientales se basan en dos principios: a) precaución y b) prevención. El primero se aplica debido al desconocimiento que se comentó anteriormente y, el segundo, cuando existe certeza de que el riesgo es inminente, por lo que debe minimizarse hasta eliminarlo.

El principio de precaución guarda una limitante en su redacción original, ya que alude a las capacidades locales sin exigir que estas deban ampliarse o mejorar ante la certeza científica de que, en otra región, se haya demostrado la existencia de riesgo por alguna práctica productiva. Mientras que el principio de prevención carece de reconocimiento consuetudinario para demandar su aplicación en favor del interés colectivo y de la justicia socioambiental, pues el contenido de esta regulación alude a la soberanía en la capacidad de aprovechamiento de la naturaleza, sin que ello implique también una responsabilidad de protección hacia sus propios ciudadanos.

De esta manera, resulta necesario valorar las implicaciones de este eje regulatorio en cuanto dichos aspectos podrían señalar que existe una seria limitante en la capacidad de los Estados para lidiar con los riesgos manufacturados de las actividades mineras y del fracking.

En este sentido, vale la pena recuperar las perspectivas de Douglas y Giddens. Por una parte, la estandarización internacional de una normativa homogénea cuya existencia se dio en el núcleo de valores europeos y estadounidenses, como son los principios de precaución y prevención respectivamente, ya que responden a elementos culturales ajenos a los que operan en otras regiones, por lo que su aplicación no solamente será deficiente, sino incluso perjudicial debido a la falta de una definición local sobre el riesgo y los alcances de este.

Los proyectos de minería y fracking, abordados en este documento, generan efectos socioambientales negativos graves, para los que la regulación local carece de mecanismos para enfrentarlos. La presencia implícita del principio de precaución en la mayoría de los casos señalados ha permitido dotar de ciertas normas para limitar los riesgos que estos ocasionan. No obstante, la debilidad institucional y la carencia de políticas ambientales integrales con este principio dificultan la ejecución de mecanismos que permitan garantizar un ambiente sano para la sociedad. 
Es evidente que este no es el único mecanismo que se puede utilizar para cumplir este derecho, pero la falta de una regulación nacional o regional surgida del debate local pone en entredicho la posibilidad institucional de que se alcance tal cuestión en el corto plazo. Es fundamental valorar la necesidad de un tratado vinculatorio que permita la discusión sobre una norma latinoamericana en este mismo sentido, la cual exija su cumplimiento, especialmente en escenarios donde actividades como la minería y el fracking amenazan a la población.

Por otra parte, las regulaciones aplicadas para los casos comentados se limitan a la prohibición o revisiones opacas del tema, por lo que carecen de marcos de trabajo integrales que busquen impedir otros problemas socioambientales. Por esta razón, aunque se restrinjan las actividades mineras o de fracking, éstas siguen ejerciéndose de forma ilegal o buscan reactivarse so pretexto de la necesidad económica y productiva.

Por lo anteriormente mencionado, la forma en que se ejecuta el principio de precaución para los casos comentados no garantiza el acceso a un ambiente sano; por el contrario, esta deteriora la situación y los riesgos socioambientales de largo plazo.

\section{Referencias}

Aidun, H. y Giunta, T. (2019). Prohibiciones y moratorias al fracking: legislación comparada. AIDA. https://aida-americas.org/sites/default/files/publication/AIDA\%20Informe_\%20Prohibiciones\%20y\%20moratorias\%20al\%20fracking.pdf

Andaluz Westreicher, C. (2006). Manual de Derecho Ambiental. Proterra. https://biblioteca.semarnat.gob.mx/janium/Documentos/Ciga/Libros2013/CD002544.pdf

Arroyo Cisneros, E. A. (2012). El derecho fundamental al medio ambiente. Porrúa.

Azamar Alonso, A. (2018). Minería en América Latina y México: problemas y consecuencias. Universidad Autónoma Metropolitana. https://www.researchgate.net/publication/331273065_ Mineria_en_America_Latina_y_Mexico_problemas_y_consecuencias

Azamar Alonso, A. y Olivera Villa, B. A. (2021). Extractivismo y desarrollo dos caras de la misma moneda: Amenaza para las mujeres en América Latina. En prensa.

Cafferatta, N. (2004). Introducción al derecho ambiental. Buenos Aires: Secretaría de Medio Ambiente y Recursos Naturales. https://cebem.org/cmsfiles/publicaciones/Introduccion_al_ Derecho_Ambiental,_Caferatta.pdf

Campanini, O., Gandarillas, M. y Gudynas, E. (2019). Derechos y violencias en los extractivismos. Extrahecciones en Bolivia y Latinoamérica. CEDIB, ODDNN, CLAES.

Comisión Mundial de Ética del Conocimiento Científico y la Tecnología (COMEST). (2005). Informe del Grupo de Expertos sobre el principio precautorio. COMEST. https://bit.ly/3nmDgJu

Douglas, M. (1987). Les études de perception du risque: un état de l’art. En Jean-Louis Fabiani y Jacques Thies (Eds.), La societe vulnerable. évaluer et maîtriser les risques (pp. 46-60). Rue d'Ulm.

Douglas, M. (1996). La aceptabilidad del riesgo según las ciencias sociales. Paidós. https://bit. ly/3lzjzh3

Farràs J., Bosch, J. L. y Torrente D. (2001). La seguridad como construcción social. Ponencia presentada en el VII Congreso Español de Sociología. http://www.jmcprl.net/publicaciones/f04/ files/3\%20SEGURIDAD\%20GASOLINERAS.PDF 
Giddens, A. (2001). Un mundo desbocado. Los efectos de la globalización en nuestras vidas. Taurus.

Kiss A. y Shelton, D. (2007). Guide to International Environmental Law. Martinus Nijhoff Publishers. https://papers.ssrn.com/sol3/papers.cfm?abstract_id=1012092

Lefeber, R. (1996). Transboundary Environmental Interference and the Origin of State Responsibility. Graham y Trotman. https://brill.com/view/title/10318

Organización de las Naciones Unidas (ONU). (1992). Declaración de Río sobre el Medio Ambiente y el Desarrollo. ONU. https://www.un.org/spanish/esa/sustdev/agenda21/riodeclaration. htm

Pérez Castellón, A., Puentes Riaño, A., Rodríguez, H. y Herrera Santoyo, H. (2016). Principio de precaución: Herramienta jurídica ante los impactos del Fracking. Fundación Heinrich Böll Aida. https://mx.boell.org/sites/default/files/publikation_aida_boell_final_20160314.pdf

Pozzi, S., López Tarabochia, M. y Gálvez, A. A. (13 de diciembre 2019). Qué países dicen sí y qué países dicen no al fracking en América Latina. El Salto. elsaltodiario.com/fracking/ fracking-en-america-latina-que-paises-dicen-si-que-paises-dicen-no-y-por-que

Romero Muñoz, A. (2017). El principio de precaución en México. Estudio de caso: Emergencia ecológica responsabilidad de la minera Buena Vista del Cobre S.A. de C.V. [Tesis de Maestría]. Benemérita Universidad Autónoma de Puebla. https://repositorioinstitucional.buap.mx/ handle/20.500.12371/485

Sands P. (2018). Principles of International Environmental Law. Cambridge University Press. https://bit.ly/32N1x3C

Svampa, M. (2019). Las fronteras del neoextractivismo en América Latina. Conflictos socioambientales, giro ecoterritorial y nuevas dependencias. CALAS, Editorial Unive RS Itaria, UCR, FLACSO, UNSAM. http://calas.lat/sites/default/files/svampa_neoextractivismo.pdf

Trouwborst, A. (2007). The Precautionary Principle in General International Law: Combating the Babylonian Confusion. Review of Eruopean Community and Internation Environmental Law, 16, 185-191. https://onlinelibrary.wiley.com/doi/abs/10.1111/j.1467-9388.2007.00553.x

Zaremberg, G. y Guarneros Meza, V. (2019). Conversing with Goliath: Participation, mobilization and repression around neoextractionist and environmental conflicts. British Academy, FLACSO México y De Montfort University. https://conversingwithgoli.wixsite.com/misitio/ datos-y-mapas

Zinn, J. (2008). Social theories of risk and uncertainty. An introduction. Massachusetts: Wiley-Blackwell. https://bit.ly/38MPwPI 